\title{
Erratum to: The all-source Green's function (ASGF) and its applications to storm surge modeling, part I: from the governing equations to the ASGF convolution
}

\author{
Zhigang Xu ${ }^{1}$
}

Published online: 16 December 2015

(C) Springer-Verlag Berlin Heidelberg 2015

Erratum to: Ocean Dynamics (2015) 65:1743-1760

DOI 10.1007/s10236-015-0893-Z

The original version of this article unfortunately contained a mistake. The phrase right below Eq. 47 should be corrected as follows:

Now, $\mathbf{G}_{\boldsymbol{c} \mathbf{L}}$ is a new convolution matrix, whose sizes are $L_{G} \times n$, where $L_{G}$ is given in Eq. (29). The number of columns of $\mathbf{G}_{c \mathbf{L}}$ is $n$, whereas the number of columns of $\mathbf{G}_{\boldsymbol{c}}$ is $N$. For the surge model and MERRA model used in this study, $N=32$, 224,425 , and $n=408,622$. $\mathbf{G}_{c \mathbf{L}}$ has 408,622 columns, a reduction by $31,968,881$ from that of $\mathbf{G}_{\boldsymbol{c}}$. This is a huge reduction, which helps greatly store the matrix and enhance the computational efficiency. Therefore, instead of Eq. (21), Eq. (45) should be used for storm surge simulations; its subscripts and the tilde sign may be dropped in the context where there is no ambiguity.

The online version of the original article can be found at http://dx.doi.org/ 10.1007/s10236-015-0893-z.

\section{Zhigang Xu}

Zhigang.Xu@dfo-mpo.gc.ca

Fisheries and Oceans Canada, Maurice Lamontagne Institute, Mont-Joli, Quebec, Canada 